\title{
Development of an innovative specimen geometry for tensile split Hopkinson tests
}

\author{
Marco Peroni ${ }^{1 *}$, Jean-François Croteau ${ }^{2}$, and Elisa Cantergiani ${ }^{2}$ \\ ${ }^{1}$ European Commission, Joint Research Centre, Ispra, Italy \\ ${ }^{2}$ I-Cube Research/Bmax, 30 boulevard de Thibaud, 31104 Toulouse, France
}

\begin{abstract}
Specimen manufacturing is one of the critical issues to solve during a dynamic material characterization, especially by mean of the Hopkinson bar technique. In particular, concerning tensile testing, the sample is generally axisymmetric with two threaded ends to directly connect it to the Hopkinson bars. In this context, this work presents the development of an innovative sample geometry and related fixtures that can be widely adopted when conventional manufacturing procedures are problematic or when there are numerous specimens. The Tensile Hopkinson Extruded Design Sample (THEDS) geometry requires properly shaped bar ends that can be obtained by wire electrical-discharge machining. The THEDS geometry has been efficiently applied to a test campaign on electron-beam welded copper and niobium specimens. The performances of the developed geometry have first been assessed using digital image correlation (DIC) to compare the results obtained at quasi-static and intermediate velocities with a standard tensile specimen geometry. Secondly, dynamic tests at up to about $1000 \mathrm{~s}^{-1}$ have been performed with a modified Hopkinson bar system at the European Commission (JRC) using the THEDS geometry.
\end{abstract}

\section{Introduction}

Specimen manufacturing is one of the critical issues to solve during a dynamic material characterization, especially by mean of the Hopkinson bar technique. In Split Hopkinson bars compression tests, the specimen geometry is typically cylindrical or at the most parallelepipedal, while several specimen geometries have been proposed for tensile testing. The different specimen designs have the same main objective: to directly connect the specimen to the Hopkinson bars without adapters to reduce additional masses that can produce fictitious oscillations in the stress-strain measurements.

The oldest specimen geometry and the most adopted in literature works is probably axisymmetric with two threaded ends as first presented in [1]. This solution minimizes the mass of the specimen and guarantees a robust connection to the equipment bars. Several numerical studies assessed the performances of this topology and provided indications to optimize it in different testing conditions and for different materials (see [2] for an example). The two main drawbacks of this configuration are related to specimen manufacturing and test operation. Firstly, the production of this specimen geometry (in general performed with

* Corresponding author: marco.peroni@ec.europa.eu 
computer numerical control (CNC) lathes) is time consuming, expensive, and requires advanced machining expertise when tested material cannot be machined with standard procedures or machining parameters due to its physical or chemical properties. Secondly, for the drawback related to test operation, specimens with threaded ends imply the rotation of the split Hopkinson bars to mount the specimen and this operation could be problematic with long bars and needs removable strain-gage connections.

Another specimen geometry largely adopted in testing of sheet materials consists of connect a standard "dogbone" geometry (usually with bolts, pins or adhesives) to two fixtures with threaded ends that ensure the connection to the equipment bars. Several experimental [3-4] and numerical studies [5-6] are available in literature and propose manufacturing processes and indications to reduce the disadvantages related to additional masses between the specimen and the Hopkinson bars. Concluding this hint concerning tensile Hopkinson specimen designs, additional geometries have been developed for large scale applications [7] or to adopt classical compressive Split Hopkinson setup to generate a tensile load into the specimen [8].

\section{Tensile Hopkinson Extruded Design Sample (THEDS)}

This work presents the development of an innovative sample geometry and related fixtures that can be widely adopted when conventional manufacturing procedures are problematic or when there are numerous specimens to test. The new design principle (inspired by the fir-tree gripping system proposed in [9]) is to create a geometry obtainable using wire discharge machining as reported in Figure 1a. In practice the cylindrical threaded-end shape has been modified in an "extruded" geometry (using 3D modelling terminology) able to transmit axial forces.
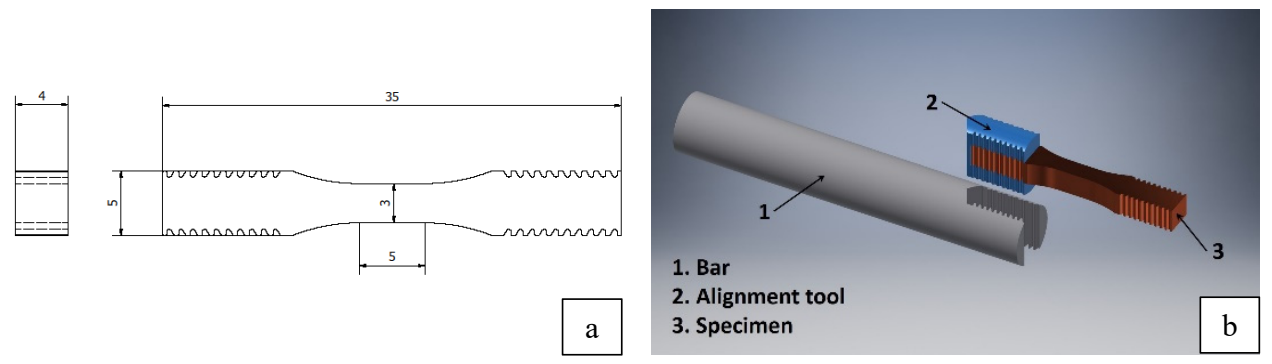

Fig. 1. a) proposed sample geometry and b) diagram of the bar-specimen assembly.

The Tensile Hopkinson Extruded Design Sample (THEDS) geometry associated with wire discharge machining has several advantages compared with the standard threaded-end specimen:

- manufacturing is totally automated, faster, and cheaper;

- the THEDS design can be applied to all metals included ones that present issues during conventional lathe machining (e.g. soft or additively manufactured metals);

- specimens can easily be extracted from plates, resulting in a rectangular cross-section;

- using proper tolerances, sample mounting is simplified in the Hopkinson bar ends because the specimen is only transversally inserted and not screwed into the bars.

On the other hand, the THEDS geometry requires properly shaped bar ends (as well as double threaded ends specimen) that can be obtained by wire electrical-discharge machining, as reported in Figure $1 \mathrm{~b}$ that presents a diagram of the sample-bar assembly. The cyan component (2) is simply an alignment tool and, therefore, does not contribute to the load transmission, unless adhesive is used. Figure 2 reports the final realization of the THEDS 
geometry with a fixtures example (Figure 2a) and the components for quasi-static/low strainrate test performed on a universal testing machine (MTS 810). In this case a proper insert has been designed to fit the MTS fixture (on the top side) and an additional dynamic load cell (on the bottom side).
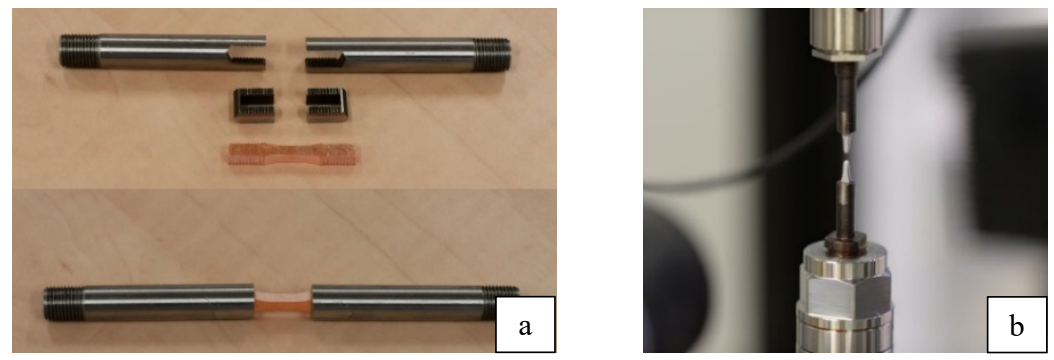

Fig. 2. a) example of the THEDS with bar fixtures and b) the THEDS specimen mounted after a static test on an MTS machine.

\section{Experimental validation of the THEDS geometry}

Finite element analysis was used to test several specimen shapes to achieve the final geometry proposed in Figure 1 with two main objectives: reduce the deformation in the clamping portion of the specimen and create a smooth transition between the extruded thread and gagelength section.

The THEDS geometry has been efficiently applied to a test campaign on electron-beam welded oxygen-free electronics (OFE) copper and high-purity niobium specimens [10]. In this study, the raw material could not be provided in cylindrical shape, making it difficult to manufacture conventional specimens with a circular cross-section. The low strength and hardness of high purity copper and niobium introduced additional issues for lathe machining of bars extracted from a plate. To evaluate the THEDS behaviour, tested specimens are treated as homogeneous because, in this context, EB welding do not dramatically affect the mechanical properties in the fusion zone and the heat affected zone (HAZ). The performances of the developed geometry have first been assessed using digital image correlation (DIC) to compare the results obtained at static and intermediate velocities with a standard tensile specimen geometry (following the ASTM E8 standard [11]). Figure 3a reports the comparison between the ASTM and THEDS geometry and the testing setup adopted to perform the experimental tests just mentioned (Figure $3 \mathrm{~b}$ ).
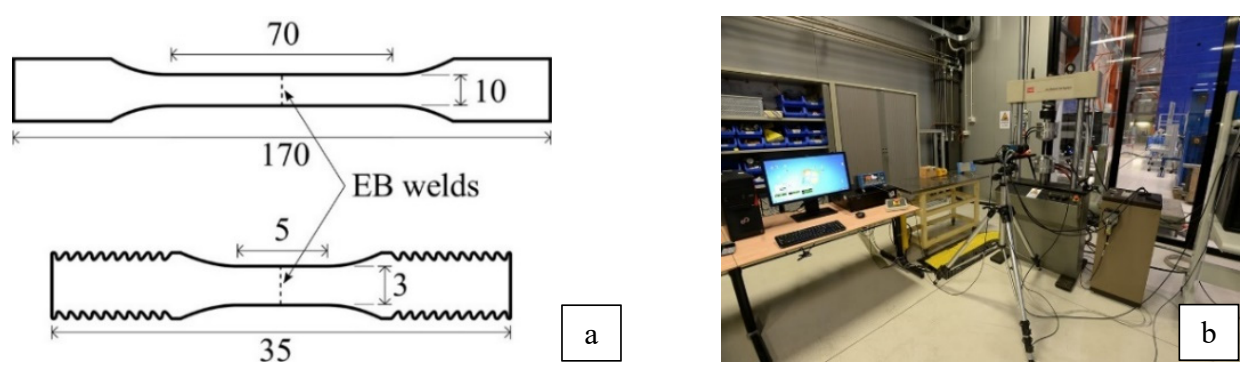

Fig. 3. a) comparison between standard tensile geometry (ASTM E8) and THEDS and b) tensile testing setup for static and intermediate velocities tests.

Validation tests have been performed with a servo-hydraulic testing machine (MTS 810) using hydraulic grips for the ASTM geometry and proper fixtures for the THEDS geometry at testing speeds corresponding to engineering strain-rates of about 0.001 and $0.1 \mathrm{~s}^{-1}$. At both 
velocities a high-resolution camera (pco.edge 5.5) was used to acquire images at frame rates of 0.5 and $50 \mathrm{fps}$, respectively, for DIC purposes (no extensometers were used due to small specimen dimensions). Figure 4a reports stress-strain (engineering) curves obtained elaborating signals recorded with the MTS machine (force and displacement) during copper tests at a strain-rate of $0.001 \mathrm{~s}^{-1}$ (similar results were measured at $0.1 \mathrm{~s}^{-1}$ and for high-purity niobium). This figure clearly shows that the two data sets (two repetitions were performed for each geometry) dramatically differ due to a wrong strain estimation in THEDS tests. This is caused by the not negligible deformation outside the gage-length that occurs for the low strength of tested material.
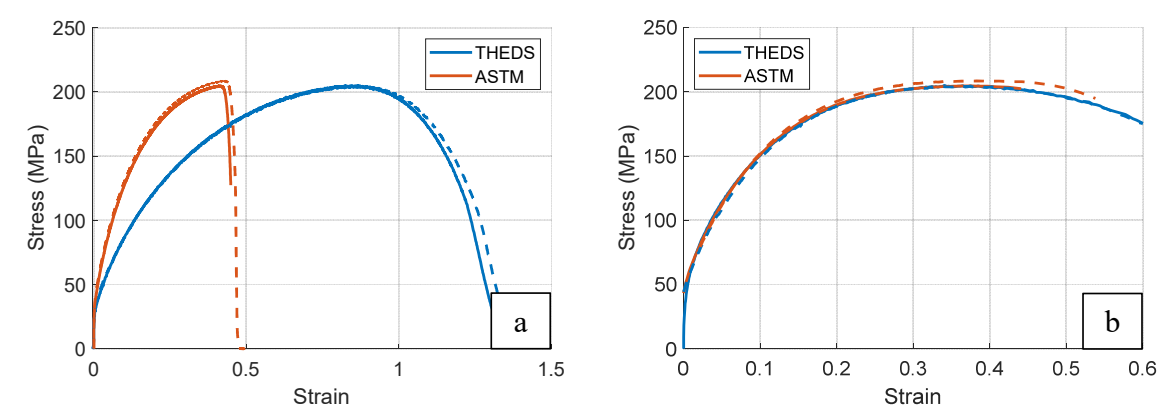

Fig. 4. Copper stress-strain (engineering) curves at $0.001 \mathrm{~s}^{-1}$ obtained elaborating a) MTS cross-head displacement and b) computing strains with DIC method.

Figure $4 \mathrm{~b}$ presents stress-strain (engineering) curves of the same tests by computing strain with 2D DIC. With this method, the curves of the two specimen geometries practically overlap. DIC data have been obtained using the MatchID software and averaging longitudinal strain over the gage-length section (subset size $15 \mathrm{px}$, step size $7 \mathrm{px}$, strain windows $15 \mathrm{px}$ ). Computed strains can be considered reliable up to necking (conventionally up to the maximum engineering stress value). Beyond this point, out-of-plane displacement affects the precision of 2D DIC and strain localizations are smoothed by the averaging procedure adopted. In any case, DIC must be used (also with traditional cylindrical double threaded end geometry) to correctly evaluate specimen strain of ductile materials when extensometer adoption cannot be used, like in split Hopkinson bars tests. In addition, the THEDS geometry provides a better estimation of Young's modulus because the grips, in this case, do not deform the specimen's ends during mounting. With hydraulics grips the applied compression induces small out-of-plane specimen bending that substantially affects Young's modulus estimation, even if the clamping force is reduced for low-strength materials.

The results reported in Figure $4 \mathrm{~b}$ could be alternatively obtained (with a reduced and questionable accuracy) by computing strains from MTS displacement signal and dividing it by a larger gage-length (compared to the actual geometrical value and preliminary evaluated with DIC or numerical simulations) and keeping into account deformation that occurs outside gage-length section. Anyway, the adoption of DIC methodology makes measurement totally independent by these "elaboration tricks" (that are commonly used in these conditions) and allows an improved estimation of material parameters (including Young's modulus if THEDS is adopted).

\section{Application of the THEDS to high strain-rate characterisation}

As previously mentioned, the THEDS was developed to investigate the high strain-rate behaviour of the two materials presented in the previous paragraph (EB welded OFE copper and high purity niobium). Results concerning the ASTM specimen geometry presented in the 
previous section to confirm the reliability of the properties measured with the THEDS geometry are not discussed in this section and the ASTM geometry was not used for the split Hopkinson bars tests. Experimental tests have been performed at five strain-rate levels of about $0.001,0.1,10,200$ and $750 \mathrm{~s}^{-1}$ using two different testing rigs. Quasi-static and intermediate strain-rate tests (up to $10 \mathrm{~s}^{-1}$ ) were performed using the setup presented in Figure $3 \mathrm{~b}$ (MTS testing machine, pco.edge 5.5 camera for quasi-static tests and IDT OS8-S3 camera for intermediate velocity tests).

Dynamic tests have been performed on a modified split Hopkinson bar with $10 \mathrm{~mm}$ diameter high strength stainless steel bars (Figure 5a) and proper THEDS fixtures at the bar ends as presented in Figure 5b. Two repetitions for each testing condition were performed (more details about specimens and equipment are available in [10]).
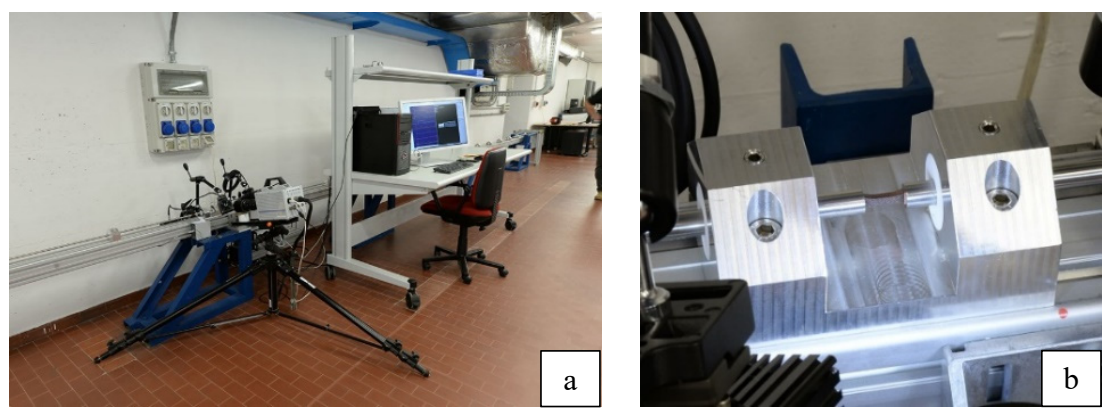

Fig. 5. a) Modified Hopkinson bar at European Commission (JRC) and b) detail of THEDS specimen and fixtures for Hopkinson bar.

Figures 6 show the stress-strain (engineering) curves for both materials tested at different strain-rates. Strains have been computed using the same DIC methodology presented in the previous section using the MatchID software package and averaging longitudinal strain along the specimen gage section.
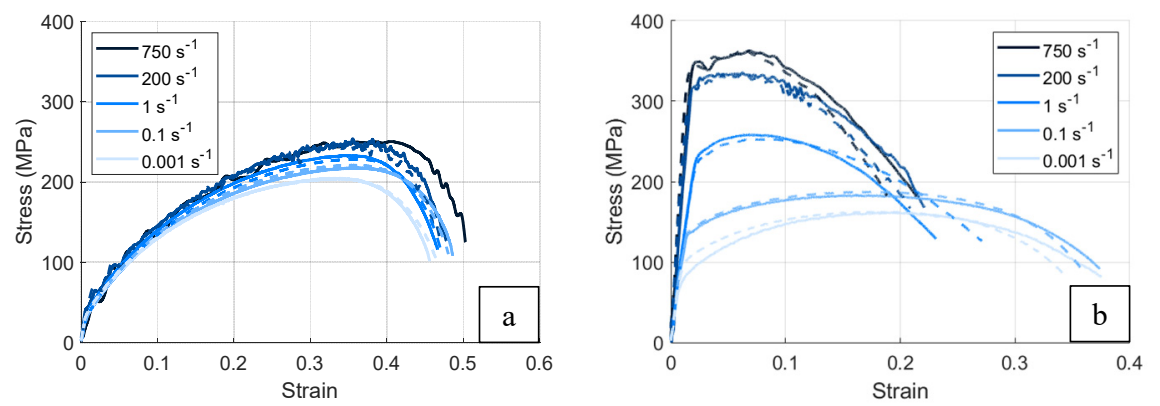

Fig. 6. Stress-strain (engineering) curves obtained at the five strain-rate levels for a) OFE copper and b) high purity niobium EB welded.

OFE copper seems slightly influenced by strain-rate in terms of yield stress and ultimate stress. On the other hand, the niobium behaviour, i.e. yield stress, strain-at-maximum-stress and strain-to-failure, is confirmed to be largely influenced by strain-rate. In addition, strain hardening decreases when increasing strain-rate. Experimental results confirm the material behaviour observed in similar tests in the literature [12] demonstrating that the THEDS design can be proficiently adopted to study the dynamic mechanical properties of metals.

In addition to stress-strain curves presented, Figure 7 shown a high speed photo sequence captured (at $50000 \mathrm{fps}$ ) during a split Hopkinson test performed at about $200 \mathrm{~s}^{-1}$ on an OFE copper THEDS. As shown no problems occurred during the tests and the bar alignment remains very satisfying. 

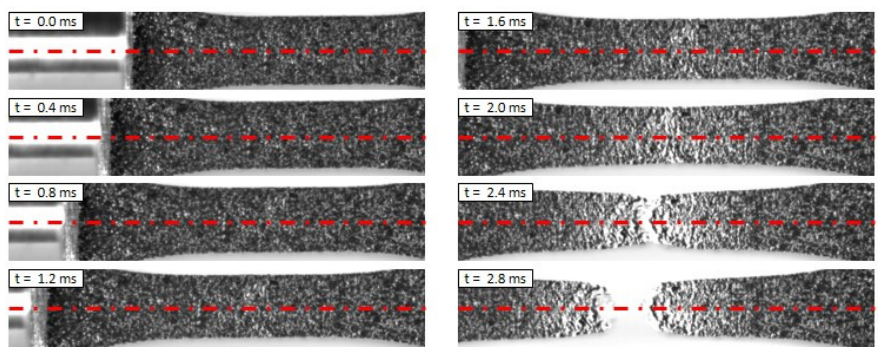

Fig. 7. High-speed photo sequence of OFE copper tested at $200 \mathrm{~s}^{-1}$.

\section{Conclusions}

In conclusion this work presents an alternative specimen geometry, named THEDS, suitable for high strain-rate metal characterisation by mean of standard testing machines and split Hopkinson bars. This geometry has been developed using FEM simulations and validated by comparing data obtained with a standard specimen geometry.

Adopting a DIC methodology, accurate strain measurements (both in quasi-static and dynamic experiments) were obtained and were independent of the deformation grip ends. The THEDS geometry has two main advantages compared to traditionally used fixtures: the reduction of manufacturing costs and complexity (especially with non-conventional metals, such as additive manufacturing samples) and the simplification of specimen mounting procedure in split Hopkinson tests. At the same time, the specimen deformation at the fixture zone remains acceptable also with low strength material as demonstrated in [10] and bar alignments is maintained during deformation. Further applications of the THEDS geometry could be to composites or plastics that can be machined with automated high precision techniques, such as waterjet or laser cutting.

The experimental data used in this research were generated through access to the ELSA HopLab under the Framework of access to the Joint Research Centre Physical Research Infrastructures of the European Commission (DWAM project, Research Infrastructure Access Agreement Nr. 35509-1/2019-1-RDELSA-HopLab).

\section{References}

1. T. Nicholas, Exp. Mech. 21, (1981)

2. D.A. Prabowo, M. A. Kariema and L. Gunawan, Procedia Engineering 173 (2017)

3. N. Ledford, H. Paul, G. Ganzenmuller, M. May, M. Hofemann, M. and Nikica Petrinic, EPJ Web of Conferences 94, (2015)

4. P. Verleysen, J. Degrieck, J. Van Slycken, B. De Cooman and L. Samek, Structures Under Shock and Impact VIII (2004)

5. P. Verleysen, B. Verhegghe, T. Verstraete and D. Joris, Lat. Am. J. Solids Struct. 6 (2009)

6. Q. Wang F. Xu, B. Yang, L. Feng, D. Huang, C. Wamg and B Wu, IEEE Access 8 (2020)

7. C.Albertini, P. Boone and M Montagnani, J. Phys. Colloq. 46 (1985)

8. D. Mohr, G. Gary Exp. Mech. 47, (2007)

9. M.P. Weyer, T.J. Cloetea and R.A. Govender, Eur. Phys. J. Special Topics 227 (2018) 
10. J.F. Croteau, M. Peroni, S. Atieh, N. Jacques and E. Cantergiani, J. dynamic behavior mater. (2021)

11. ASTM International, ASTM E8 / E8M-16ae1, Standard Test Methods for Tension Testing of Metallic Materials (2016)

12. L. Peroni and M. Scapin, EPJ Web Conf. 183 (2018) 